\title{
Corneal confocal microscopy for assessment of diabetic peripheral neuropathy: a meta-analysis
}

\author{
Min-Shan Jiang, ${ }^{1,2}$ Ying Yuan, ${ }^{3}$ Zhao-Xiang Gu, ${ }^{4}$ Song-Lin Zhuang ${ }^{1}$
}

\begin{abstract}
- Additional material is published online only. To view please visit the journal online (http://dx.doi.org/10.1136/ bjophthalmol-2014-306038).

${ }^{1}$ Shanghai Key Lab of Modern Optical Systems, Engineering Research Center of Optical Instruments and Systems, Ministry of Education, University of Shanghai for Science and Technology, Shanghai, China ${ }^{2}$ Department of Biomedical Engineering, Florida International University, Miami, Florida, USA

${ }^{3}$ Department of Radiology, Shanghai Ninth People's Hospital, Affiliated to Shanghai JiaoTong University School of Medicine, Shanghai, China ${ }^{4}$ Department of Nuclear Medicine, Huadong Hospital, Fudan University, Shanghai, China
\end{abstract}

\section{Correspondence to} Dr Ying Yuan, Department of Radiology, Shanghai Ninth People's Hospital, Affiliated to Shanghai JiaoTong University School of Medicine, 639 Zhizaoju Road, Shanghai 200011, China; yuany83@163.com

Received 21 August 2014 Revised 5 January 2015 Accepted 25 January 2015 Published Online First 12 February 2015

\section{CrossMark}

\begin{tabular}{l}
\hline To cite: Jiang M-S, Yuan Y, \\
Gu Z-X, et al. Br J \\
Ophthalmol 2016;100: \\
9-14. \\
\hline
\end{tabular}

Ophthalmol 2016:100

9-14.

\begin{abstract}
Purpose To evaluate the diagnostic performance of corneal confocal microscopy (CCM) in assessing corneal nerve parameters in patients with diabetic peripheral neuropathy (DPN).
\end{abstract}

Methods Studies in the literature that focused on CCM and DPN were retrieved by searching PubMed, Excerpt Medica Database (EMBASE) and China National Knowledge Infrastructure (CNKI) databases. RevMan V.5.3 software was used for the meta-analysis. The results are presented as weighted mean difference (WMD) with a corresponding 95\% Cl.

Results 13 studies with a total of 1680 participants were included in the meta-analysis. The pooled results showed that the corneal nerve fibre density, nerve branch density and nerve fibre length were significantly reduced (all $p<0.00001$ ) in the patients with DPN compared with healthy controls ((WMD $=-18.07,95 \%$ $\mathrm{Cl}-21.93$ to -14.20$)$, (WMD $=-25.35,95 \% \mathrm{Cl}$ -30.96 to -19.74$)$ and $(\mathrm{WMD}=-6.37,95 \% \mathrm{Cl}-7.44$ to -5.30$))$ and compared with the diabetic patients without DPN ((WMD $=-8.83,95 \% \mathrm{Cl}-11.49$ to -6.17), (WMD $=-13.54,95 \% \mathrm{Cl}-20.41$ to -6.66$)$ and (WMD $=-4.19,95 \% \mathrm{Cl}-5.35$ to -3.04$))$, respectively. No significant difference was found in the corneal nerve fibre tortuosity coefficient between diabetic patients with DPN and healthy controls $(p=0.80)$ or diabetic patients without DPN ( $p=0.61)$.

Conclusions This meta-analysis suggested that CCM may be valuable for detecting and assessing early nerve damage in DPN patients.

\section{INTRODUCTION}

Diabetic neuropathy is heterogeneous, affecting different parts of the nervous system and resulting in diverse clinical symptoms. Reappraisal of diabetic neuropathy definitions and diagnostic criteria has been recently provided by the Toronto Diabetic Neuropathy Expert Group. ${ }^{1}$ The expert panel proposed definitions for typical diabetic peripheral neuropathy (DPN) as a chronic, symmetrical, length-dependent diabetic sensorimotor polyneuropathy. Early and accurate detection of DPN enables prevention of long-term complications, anticipation of deterioration and assessment of new therapies. Symptom scores, quantitative sensory testing and electrophysiology are some of the diagnostic tools used to identify DPN. However, current methods are unsatisfactory in that they lack sensitivity and require expert assessment, focusing only on large fibres or employing invasive methodology. ${ }^{1}$

Corneal confocal microscopy (CCM) is a noninvasive method that allows study of the human cornea in vivo. It has increasingly been used to assess the morphology of the sub-basal corneal nerve plexus. The main parameters of corneal nerve morphology include nerve fibre density (NFD), nerve branch density (NBD), nerve fibre length (NFL) and corneal nerve fibre tortuosity or the tortuosity coefficient (TC). Several review articles have described the principles, applications and practical approaches to capture images, and the clinical correlation of CCM in the study of corneal nerves in diabetic and other peripheral neuropathies. ${ }^{2-4}$ Recent studies have assessed the diagnosis and monitoring of DPN using CCM. $^{5-19}$ However, controversial results exist and some studies are less convincing because of a small sample size. Therefore, a meta-analysis is imperative because it is a powerful tool for summarising results from different studies by producing a single estimate of the major effect with enhanced accuracy. The purpose of the current study was to perform a systematic review and meta-analysis to evaluate the diagnostic performance of CCM in assessing corneal nerve parameters in patients with DPN, when compared with healthy controls and diabetic patients without DPN.

\section{METHODS}

The process of the meta-analysis was performed according to the Cochrane Collaboration recommendations. ${ }^{20}$ The analysis results were reported according to the Preferred Reporting Items for Systematic Reviews and Meta-Analyses (PRISMA) statement. $^{21}$

\section{Identification and eligibility of relevant studies}

A systematic search was performed using PubMed, Excerpt Medica Database (EMBASE) and China

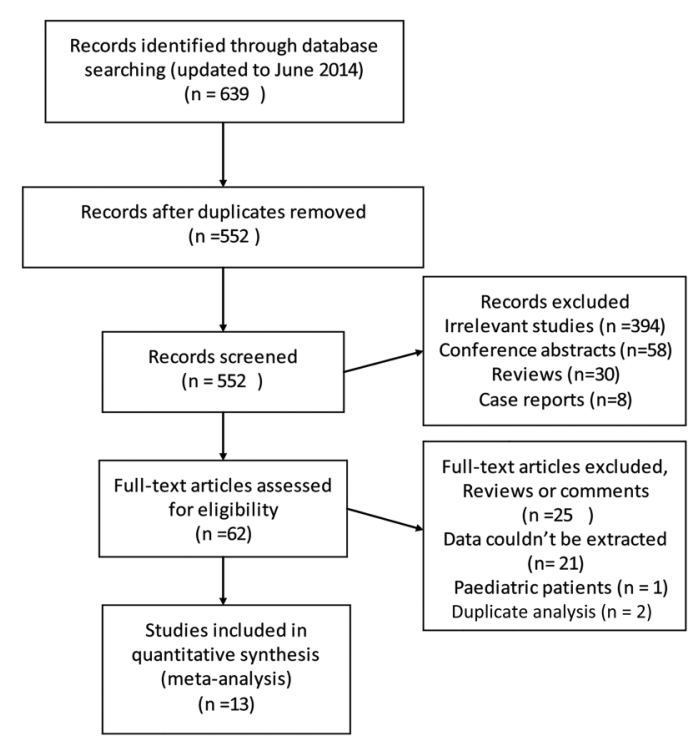

Figure 1 Flow diagram of article selection. 
National Knowledge Infrastructure (CNKI) to identify abstracts of articles involving human subjects (updated to June 2014). The search strategy was based on a combination of terms: (1) "corneal confocal microscopy"; (2) "diabetes" or "diabetic" and (3) "neuropathy" or "neuropathies". The following inclusion criteria were adopted: (1) published between January 2003 and June 2014; (2) including at least 10 adult patients with DPN; (3) study subjects: the disease group included patients with DPN who met the international diagnostic criteria for $\mathrm{DPN}^{1}$ and (4) at least one corneal nerve parameter (NFD, NBD, NFL or TC) was used for detection of CCM. Exclusion criteria included: abstracts from conferences and full texts without raw data available for retrieval, case reports, comments and reviews. When multiple publications from the same study population were available, the most recent publication was included.
Data extraction and risk of bias assessment

Two reviewers extracted information from the eligible articles. Extracted data included study details (such as the first author's name, year of publication, glycated haemoglobin $\left(\mathrm{HbA}_{1 \mathrm{c}}\right)$, corneal nerve parameters, type of CCM and exclusion criteria) and patient characteristics (such as mean age, gender of patients, diabetes type, duration of diabetes and sample size). The risk of bias was assessed based on guidance provided in the Cochrane Handbook for Systematic Reviews of Interventions. ${ }^{20}$ Studies were not excluded from the meta-analysis based on a high risk of bias, but the risk of bias was taken into consideration during interpretation of the results.

\section{Statistical analysis}

Review Manager V.5.3 (RevMan) was used for the meta-analysis. Published data of cross-sectional studies for corneal nerve

Table 1 Characteristics of included studies

\begin{tabular}{|c|c|c|c|c|c|c|c|c|c|c|c|c|c|}
\hline \multirow[b]{2}{*}{ Study } & \multirow[b]{2}{*}{ Groups } & \multirow[b]{2}{*}{$\mathrm{N}$} & \multirow{2}{*}{$\begin{array}{l}\text { Age } \\
\text { (years) }\end{array}$} & \multirow{2}{*}{$\begin{array}{l}\text { Sex } \\
\text { (M/F) }\end{array}$} & \multirow{2}{*}{$\begin{array}{l}\text { Diabetes } \\
\text { type }(1 / 2)\end{array}$} & \multirow{2}{*}{$\begin{array}{l}\text { Duration of } \\
\text { diabetes (years) }\end{array}$} & \multirow{2}{*}{$\begin{array}{l}\mathrm{HbA}_{1 \mathrm{c}} \\
(\%)\end{array}$} & \multirow{2}{*}{$\begin{array}{l}\text { Exclusion } \\
\text { criteria* }\end{array}$} & \multirow{2}{*}{$\begin{array}{l}\text { Type } \\
\text { of CCM }\end{array}$} & \multicolumn{4}{|c|}{ Assessment with CCM } \\
\hline & & & & & & & & & & NFD & NBD & NFL & TC \\
\hline \multirow[t]{3}{*}{ Ahmed et $a l^{5}$} & Controls & 64 & $38.9 \pm 17.6$ & $30 / 34$ & - & - & $5.5 \pm 0.4$ & $\sqrt{ }$ & LSCM & $\sqrt{ }$ & $\sqrt{ }$ & $\sqrt{ }$ & $\sqrt{ }$ \\
\hline & No-DPN & 56 & $34.9 \pm 14.8$ & $27 / 29$ & $56 / 0$ & $17.6 \pm 14.0$ & $7.4 \pm 1.3$ & $\sqrt{ }$ & & $\sqrt{ }$ & $\sqrt{ }$ & $\sqrt{ }$ & $\sqrt{ }$ \\
\hline & DPN & 33 & $50.0 \pm 14.3$ & $16 / 17$ & $33 / 0$ & $31.4 \pm 13.5$ & $8.7 \pm 2.1$ & $\sqrt{ }$ & & $\sqrt{ }$ & $\sqrt{ }$ & $\sqrt{ }$ & $\sqrt{ }$ \\
\hline \multirow[t]{3}{*}{ Edwards et al ${ }^{6}$} & Controls & 61 & $52 \pm 14$ & $27 / 34$ & - & - & $5.4 \pm 0.3$ & $\sqrt{ }$ & LSCM & & $\sqrt{ }$ & $\sqrt{ }$ & $\sqrt{ }$ \\
\hline & No-DPN & 143 & $48 \pm 16$ & $66 / 77$ & $105 / 38$ & $14 \pm 12$ & $7.8 \pm 1.2$ & $\sqrt{ }$ & & & $\sqrt{ }$ & $\sqrt{ }$ & $\sqrt{ }$ \\
\hline & DPN & 88 & $58 \pm 9$ & $57 / 31$ & $51 / 37$ & $23 \pm 14$ & $8.2 \pm 1.7$ & $\sqrt{ }$ & & & $\sqrt{ }$ & $\sqrt{ }$ & $\sqrt{ }$ \\
\hline \multirow[t]{3}{*}{ Hertz et $a l^{7}$} & Controls & 20 & $41.4 \pm 17.3$ & $5 / 15$ & - & - & $5.5 \pm 0.4$ & N/A & LSCM & $\sqrt{ }$ & $\sqrt{ }$ & $\sqrt{ }$ & $\sqrt{ }$ \\
\hline & No-DPN & 12 & $42.8 \pm 16.9$ & $18 / 8$ & $26 / 0$ & $22.7 \pm 16.4$ & $8.0 \pm 1.9$ & N/A & & $\sqrt{ }$ & $\sqrt{ }$ & $\sqrt{ }$ & $\sqrt{ }$ \\
\hline & DPN & 14 & & & & & & N/A & & $\sqrt{ }$ & $\sqrt{ }$ & $\sqrt{ }$ & $\sqrt{ }$ \\
\hline \multirow[t]{2}{*}{ Kallinikos et $a l^{8}$} & Controls & 18 & $57.8 \pm 11.5$ & $\mathrm{~N} / \mathrm{A}$ & - & - & $<6.5$ & N/A & SSCM & & & & $\sqrt{ }$ \\
\hline & DPN & 18 & $57.8 \pm 12.8$ & $\mathrm{~N} / \mathrm{A}$ & $7 / 11$ & $22.9 \pm 6.3$ & $8.1 \pm 1.2$ & $\sqrt{ }$ & & & & & $\sqrt{ }$ \\
\hline \multirow[t]{2}{*}{ Malik et $a l^{9}$} & Controls & 18 & $57.8 \pm 11.5$ & $\mathrm{~N} / \mathrm{A}$ & - & - & $<6.5$ & N/A & SSCM & $\sqrt{ }$ & $\sqrt{ }$ & $\sqrt{ }$ & \\
\hline & DPN & 18 & $57.8 \pm 12.8$ & $\mathrm{~N} / \mathrm{A}$ & $7 / 11$ & $22.9 \pm 6.3$ & $8.1 \pm 1.2$ & $\sqrt{ }$ & & $\sqrt{ }$ & $\sqrt{ }$ & $\sqrt{ }$ & \\
\hline \multirow[t]{2}{*}{ Mehra et $a l^{10}$} & Controls & 15 & $46 \pm 3$ & $\mathrm{~N} / \mathrm{A}$ & - & - & N/A & N/A & SSCM & $\sqrt{ }$ & $\sqrt{ }$ & $\sqrt{ }$ & $\sqrt{ }$ \\
\hline & DPN & 20 & $41 \pm 1$ & $\mathrm{~N} / \mathrm{A}$ & $20 / 0$ & $27 \pm 2$ & $8.9 \pm 1.4$ & N/A & & $\sqrt{ }$ & $\sqrt{ }$ & $\sqrt{ }$ & $\sqrt{ }$ \\
\hline \multirow[t]{3}{*}{ Petropoulos et al ${ }^{11}$} & Controls & 55 & $51.7 \pm 11.4$ & $28 / 27$ & - & - & $5.5 \pm 0.3$ & $\sqrt{ }$ & LSCM & $\sqrt{ }$ & $\sqrt{ }$ & $\sqrt{ }$ & \\
\hline & No-DPN & 86 & $50.4 \pm 14.1$ & $108 / 78$ & $\mathrm{~N} / \mathrm{A}$ & $24.2 \pm 21.2$ & $7.7 \pm 1.6$ & $\sqrt{ }$ & & $\sqrt{ }$ & $\sqrt{ }$ & $\sqrt{ }$ & \\
\hline & DPN & 100 & & & N/A & $34.4 \pm 17.3$ & $7.9 \pm 1.6$ & $\sqrt{ }$ & & $\sqrt{ }$ & $\sqrt{ }$ & $\sqrt{ }$ & \\
\hline \multirow[t]{3}{*}{ Pritchard et $a l^{12}$} & Controls & 154 & $46 \pm 15$ & $70 / 84$ & - & - & $5.5 \pm 0.3$ & $\sqrt{ }$ & LSCM & & $\sqrt{ }$ & $\sqrt{ }$ & \\
\hline & No-DPN & 168 & $43 \pm 16$ & $85 / 83$ & $168 / 0$ & $20 \pm 15$ & $8.0 \pm 1.2$ & $\sqrt{ }$ & & & $\sqrt{ }$ & $\sqrt{ }$ & \\
\hline & DPN & 74 & $57 \pm 11$ & $41 / 33$ & $74 / 0$ & $34 \pm 16$ & $8.6 \pm 1.8$ & $\sqrt{ }$ & & & $\sqrt{ }$ & $\sqrt{ }$ & \\
\hline \multirow[t]{3}{*}{ Quattrini et $a l^{13}$} & Controls & 15 & $55.0 \pm 18.5$ & $6 / 9$ & - & - & - & $\sqrt{ }$ & SSCM & $\sqrt{ }$ & $\sqrt{ }$ & $\sqrt{ }$ & \\
\hline & No-DPN & 10 & $53.5 \pm 10.2$ & $6 / 4$ & $3 / 7$ & $16.7 \pm 14.0$ & $7.2 \pm 1.3$ & $\sqrt{ }$ & & $\sqrt{ }$ & $\sqrt{ }$ & $\sqrt{ }$ & \\
\hline & DPN & 44 & $59.1 \pm 11.0$ & $36 / 8$ & $13 / 31$ & $21.2 \pm 12.1$ & $8.0 \pm 1.8$ & $\sqrt{ }$ & & $\sqrt{ }$ & $\sqrt{ }$ & $\sqrt{ }$ & \\
\hline \multirow[t]{2}{*}{ Sivaskandarajah et al ${ }^{14}$} & $\begin{array}{l}\text { Controls } \\
\text { No-DPN }\end{array}$ & $\begin{array}{l}64 \\
63\end{array}$ & $\begin{array}{l}38.3 \pm 16.4 \\
32.7 \pm 13.6\end{array}$ & $\begin{array}{l}34 / 30 \\
29 / 34\end{array}$ & 63/0 & $\overline{-}-17.3 \pm 12.2$ & $\begin{array}{l}5.6 \pm 0.4 \\
7.5 \pm 1.2\end{array}$ & $\sqrt{V}$ & LSCM & $\sqrt{V}$ & $\sqrt[V]{V}$ & $\sqrt{V}$ & $\sqrt{V}$ \\
\hline & DPN & 33 & $48.5 \pm 13.7$ & $14 / 19$ & $33 / 0$ & $32.3 \pm 13.1$ & $8.4 \pm 1.6$ & $\sqrt{ }$ & & $\sqrt{ }$ & $\sqrt{ }$ & $\sqrt{ }$ & $\sqrt{ }$ \\
\hline \multirow[t]{3}{*}{ Tavakoli et $a l^{15}$} & Controls & 17 & $55 \pm 19.8$ & $8 / 9$ & - & - & $<6.5$ & $\sqrt{ }$ & SSCM & $\sqrt{ }$ & $\sqrt{ }$ & $\sqrt{ }$ & \\
\hline & No-DPN & 34 & $55 \pm 11.1$ & $19 / 15$ & $2 / 32$ & $10.7 \pm 10.6$ & $8.1 \pm 1.2$ & $\sqrt{ }$ & & $\sqrt{ }$ & $\sqrt{ }$ & $\sqrt{ }$ & \\
\hline & DPN & 67 & $58.9 \pm 11.3$ & $54 / 13$ & $15 / 52$ & $17.0 \pm 12.3$ & $8.1 \pm 1.4$ & $\sqrt{ }$ & & $\sqrt{ }$ & $\sqrt{ }$ & $\sqrt{ }$ & \\
\hline \multirow[t]{2}{*}{ Tavakoli et al ${ }^{16}$} & Controls & 18 & $57.0 \pm 12.7$ & $10 / 8$ & - & - & $5.7 \pm 0.4$ & $\sqrt{ }$ & SSCM & $\sqrt{ }$ & $\sqrt{ }$ & $\sqrt{ }$ & $\sqrt{ }$ \\
\hline & DPN & 25 & $52.0 \pm 10.0$ & $20 / 5$ & $15 / 10$ & $26.5 \pm 12.5$ & $8.1 \pm 1.5$ & $\sqrt{ }$ & & $\sqrt{ }$ & $\sqrt{ }$ & $\sqrt{ }$ & $\sqrt{ }$ \\
\hline \multirow[t]{3}{*}{ Xue et $a l^{17}$} & Controls & 10 & $48.6 \pm 7.8$ & $5 / 5$ & - & - & - & N/A & LSCM & & $\sqrt{ }$ & $\sqrt{ }$ & $\sqrt{ }$ \\
\hline & No-DPN & 20 & $56.3 \pm 8.9$ & $12 / 8$ & $0 / 20$ & $8.4 \pm 5.1$ & $7.1 \pm 1.3$ & N/A & & & $\sqrt{ }$ & $\sqrt{ }$ & $\sqrt{ }$ \\
\hline & DPN & 25 & $52.0 \pm 10.6$ & $15 / 10$ & $0 / 25$ & $9.2 \pm 6.7$ & $7.9 \pm 1.6$ & N/A & & & $\sqrt{ }$ & $\sqrt{ }$ & $\sqrt{ }$ \\
\hline
\end{tabular}


parameters (mean and SD) were entered as a continuous variable. Baseline data were adopted for longitudinal studies. For studies that only reported the SE or SEM, SD was calculated with the formula $S D=S E \times \sqrt{n}$, where $\mathrm{n}$ is the sample size. In one study, ${ }^{6}$ the results were only presented in figures and data were obtained by measuring directly from these figures that were printed on a large scale. We performed three groups of comparisons for the parameters: (1) subjects with DPN versus healthy controls; (2) subjects with DPN versus control diabetic subjects without DPN (no-DPN) and (3) no-DPN versus healthy controls.

For continuous variable outcomes, the weighted mean difference (WMD) and 95\% CI were calculated. Heterogeneity between the results of different studies was examined using the $\mathrm{I}^{2}$ value, and $\mathrm{p}<0.05$ and $\mathrm{I}^{2}>50 \%$ were considered to indicate statistically significant heterogeneity. If the included studies were not heterogeneous, the fixed-effects model was used for the analysis, otherwise a random-effects model was chosen. Metaregression and subgroup analyses were performed to explain the heterogeneity across studies. A sensitivity analysis was used to determine the stability and reliability of the results. Publication bias was assessed with a funnel plot.

\section{RESULTS}

\section{Eligible studies and study characteristics}

A flow chart of the included and excluded studies is shown in figure 1. Our search strategy identified 639 potential reports. After removal of duplicate records, 552 records remained and the abstracts were reviewed based on predefined eligibility criteria. A total of 62 records were considered as potentially relevant and full texts were retrieved. At review of the full texts, 49 studies were excluded due to (1) the report was a review or comment $(n=25)$; (2) the study did not include DPN patients or outcome data were unable to be extracted $(n=21)$; (3) the study included paediatric patients with DPN $(n=1)$ and $(4)$ the articles of Tavakoli et $a l^{18}$ and Petropoulos et $a l^{19}$ overlapped with other studies. ${ }^{6} 11$ The latter two reports were viewed as eligible for including larger groups of patients or being the latest reports $(n=2)$. Ultimately, 13 studies with a total of 1680 participants (DPN 559, controls 529 and no-DPN 592) were included. Table 1 summarises the main characteristics of all eligible studies. ${ }^{5-17}$ In nine included studies, ${ }^{7-11} 1^{13}{ }^{15-17}$ diabetic patients and control subjects were matched for age.

\section{Risk of bias}

To assess selection bias, reported procedures on sequence generation and allocation concealment were judged for all studies. Often randomisation and concealment were not applicable in a study because only one experimental group was investigated. Seven studies reported the investigators interpreting the image and making the measurements of CCM images were masked to the status of the study participants (DPN, no-DPN or control). . $^{9-12} \quad 15 \quad 16$ Finally, all studies were also judged for other possible sources of bias. Some of the procedures performed during data extraction (eg, obtaining values from figures or converting SE to SD) were considered to not cause bias, but

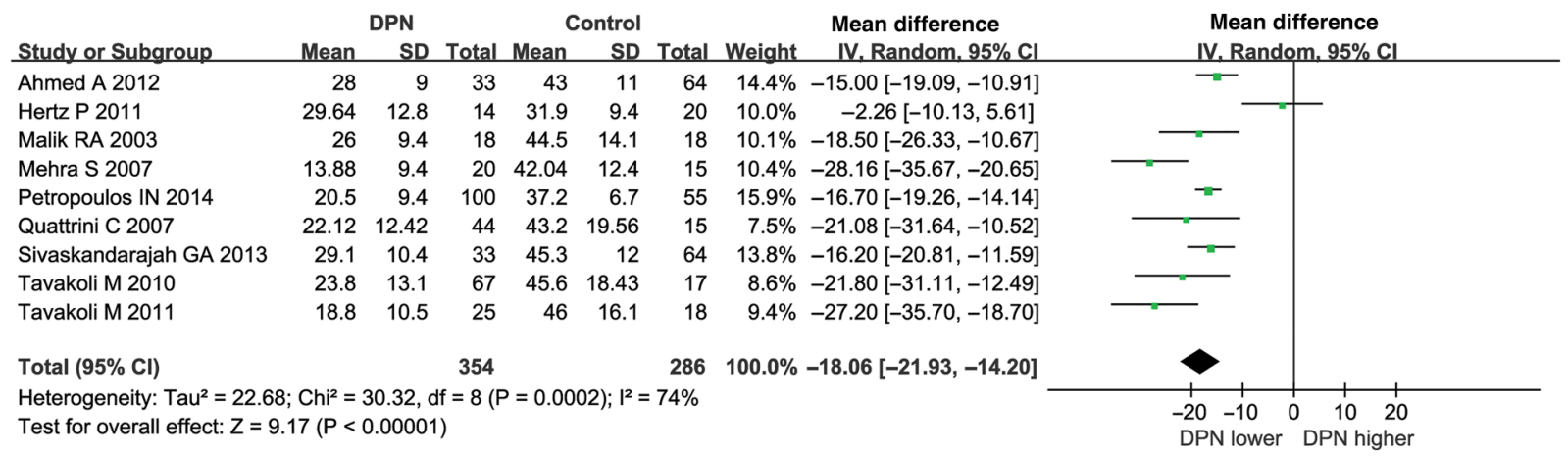

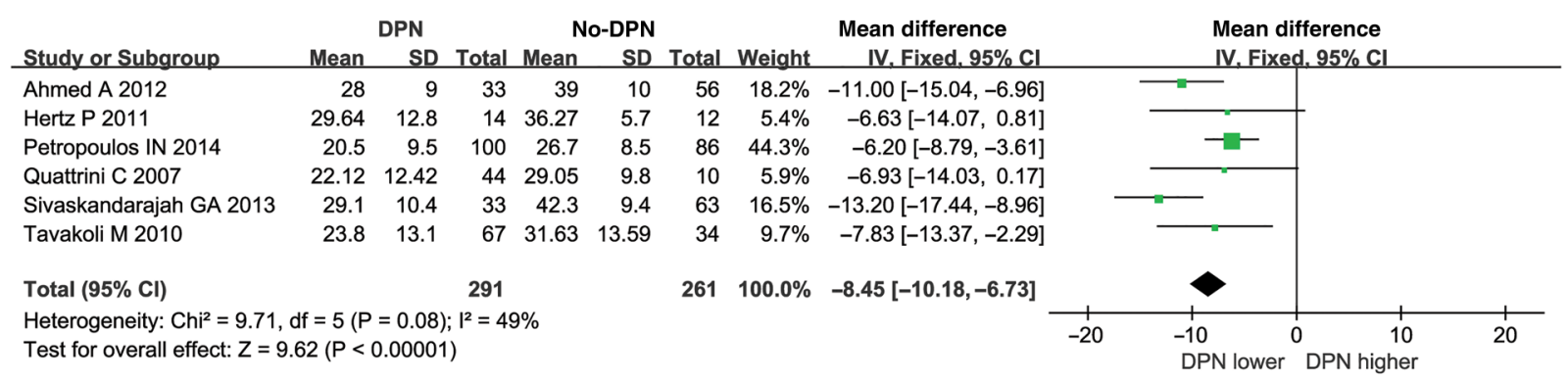

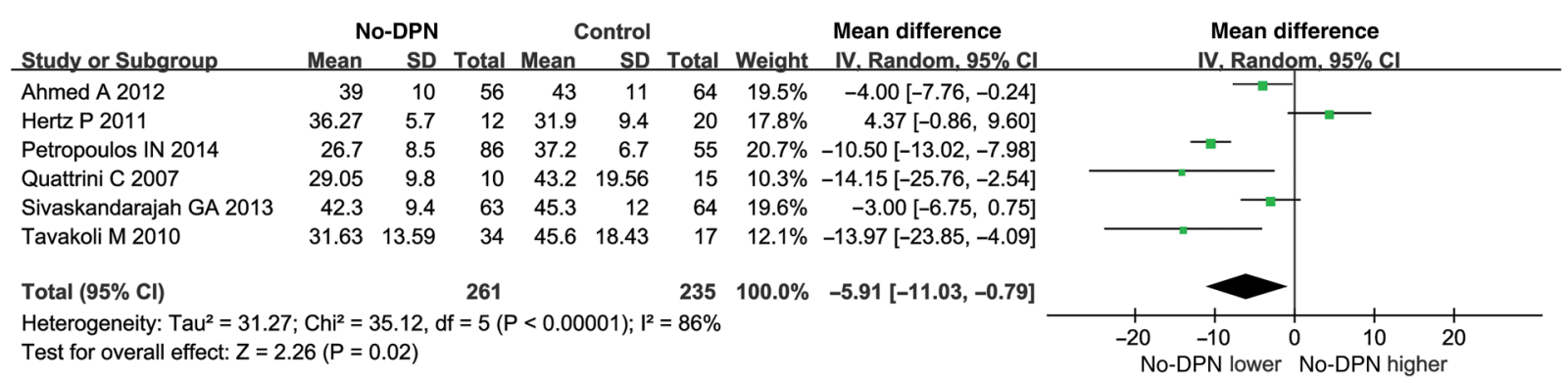

Figure 2 Forest plots of nerve fibre density (NFD) test results in diabetic peripheral neuropathy (DPN), healthy control and No-DPN groups. 
they could potentially cause some inaccuracy in the data used for the analyses. The overall risk of bias for the outcomes of interest was moderate.

\section{Data synthesis and the meta-analysis}

Nerve fibre density

Nine studies ${ }^{5}$ 9-11 13-16 with a total of 901 participants (DPN 354 , controls 286 and no-DPN 261) were included in the meta-analysis. The NFD (no. $/ \mathrm{mm}^{2}$ ) in DPN was significantly lower than the controls (WMD $=-18.07,95 \%$ CI -21.93 to $-14.20, \mathrm{p}<0.00001$ ) and no-DPN (WMD $=-8.83$, 95\% CI -11.49 to $-6.17, \mathrm{p}<0.00001)$. The detailed results are depicted in figure 2.

Nerve branch density

Twelve studies ${ }^{5-7}$ 9-17 with a total of 1644 participants (DPN 541, controls 511 and no-DPN 592) were included in the meta-analysis. The NBD (no. $/ \mathrm{mm}^{2}$ ) in DPN was significantly lower than the controls (WMD $=-25.35$, 95\% CI -30.96 to
-19.74), $\mathrm{p}<0.00001)$ and no-DPN (WMD $=-13.54,95 \% \mathrm{CI}$ -20.41 to $-6.66, \mathrm{p}<0.00001)$. The detailed results are depicted in figure 3.

Nerve fibre length

Twelve 5-7 $^{5-17}$ studies with a total of 1644 participants (DPN 541, controls 511 and no-DPN 592) were included in the meta-analysis. The NFL $\left(\mathrm{mm} / \mathrm{mm}^{2}\right)$ in DPN was significantly lower than the controls (WMD $=-6.37,95 \%$ CI -7.44 to $-5.30, \mathrm{p}<0.00001)$ and no-DPN (WMD $=-4.19$, 95\% CI -5.35 to $-3.04, \mathrm{p}<0.00001)$. The detailed results are depicted in figure 4.

Tortuosity coefficient

Eight studies ${ }^{5-8} \quad 10 \quad 14 \quad 16 \quad 17$ with a total of 875 participants (DPN 311, controls 270 and no-DPN 294) were included in the meta-analysis. There were no statistically significant differences in TCs (unitless) in the DPN both compared with the control group $(\mathrm{p}=0.80)$ and no-DPN group $(\mathrm{p}=0.61)$.

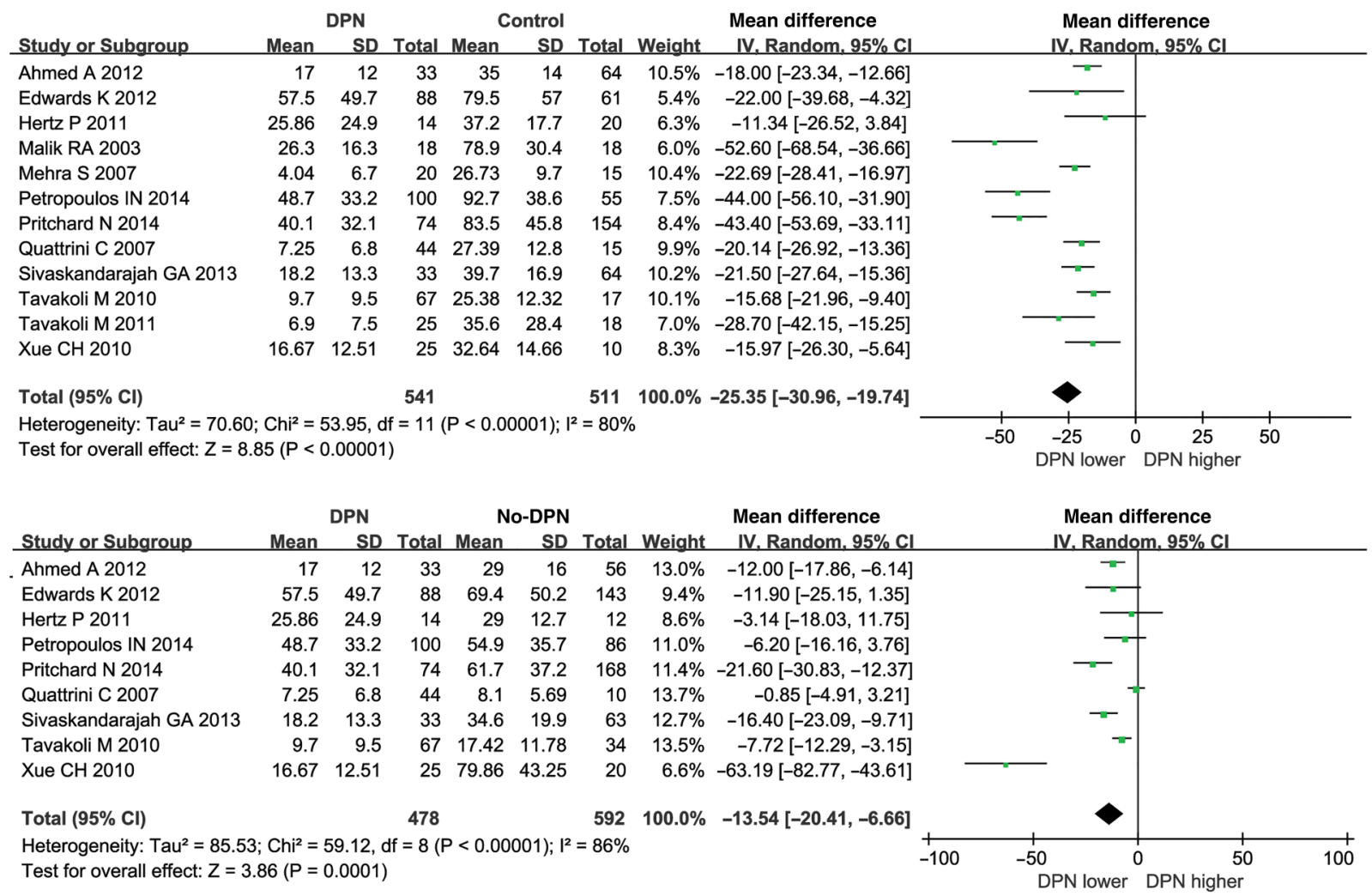

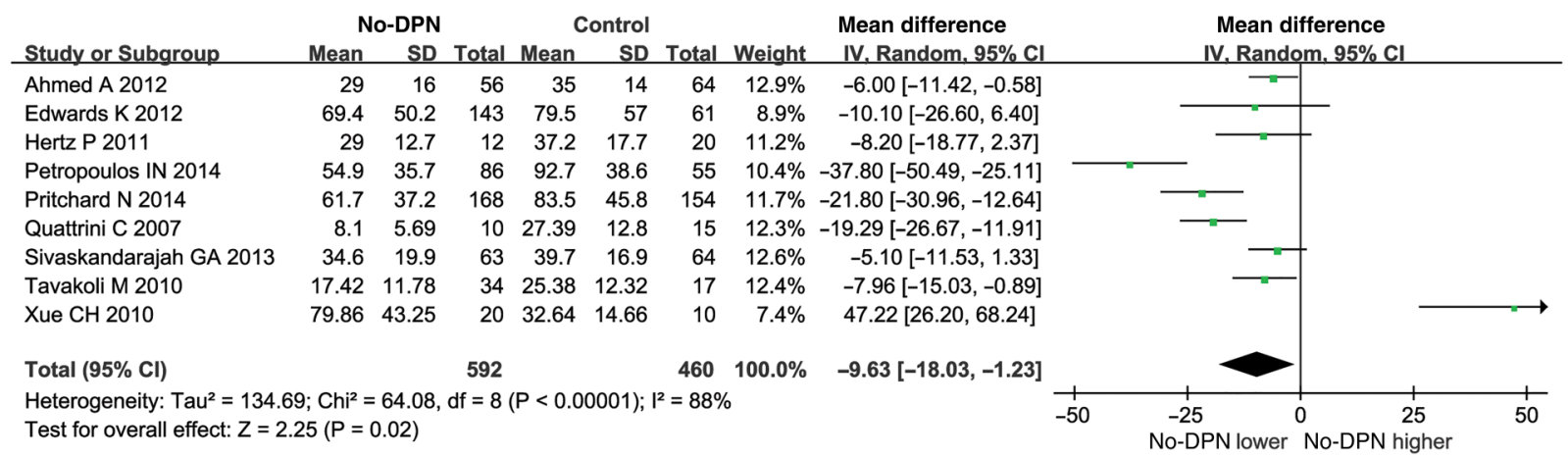

Figure 3 Forest plots of nerve branch density (NBD) test results in diabetic peripheral neuropathy (DPN), healthy control and no-DPN groups. 


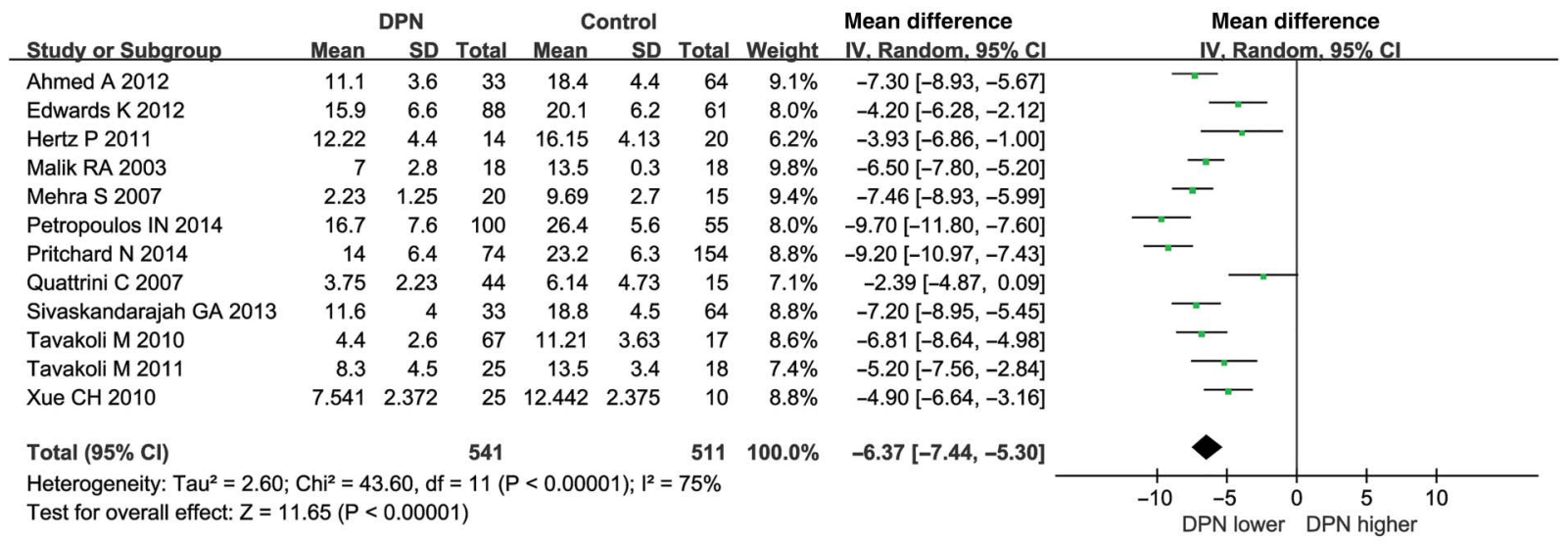

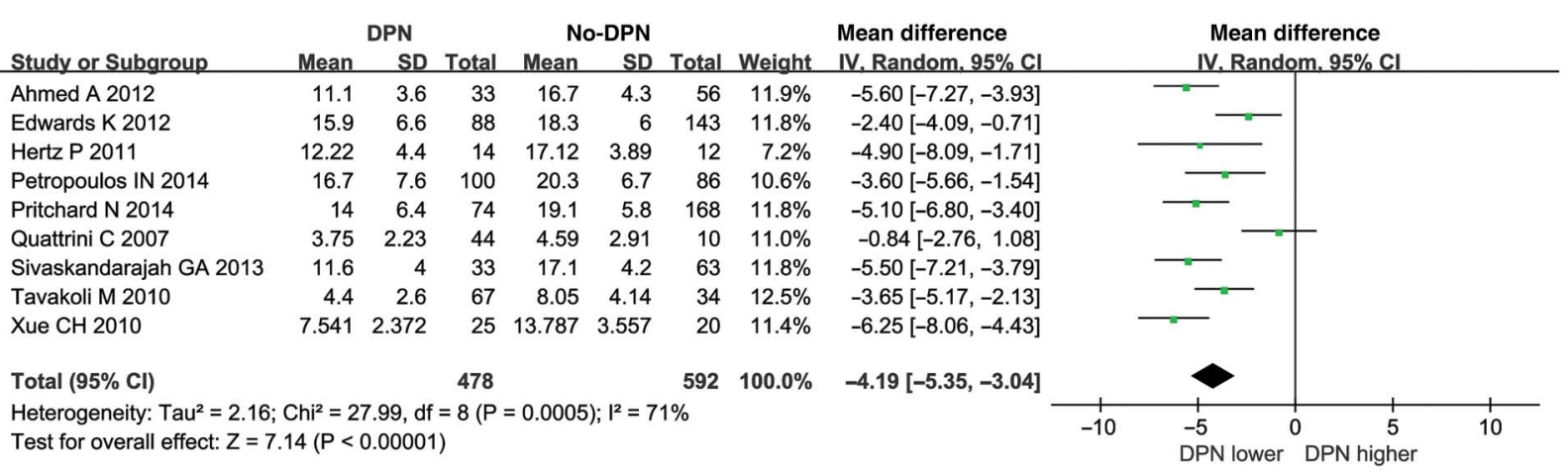

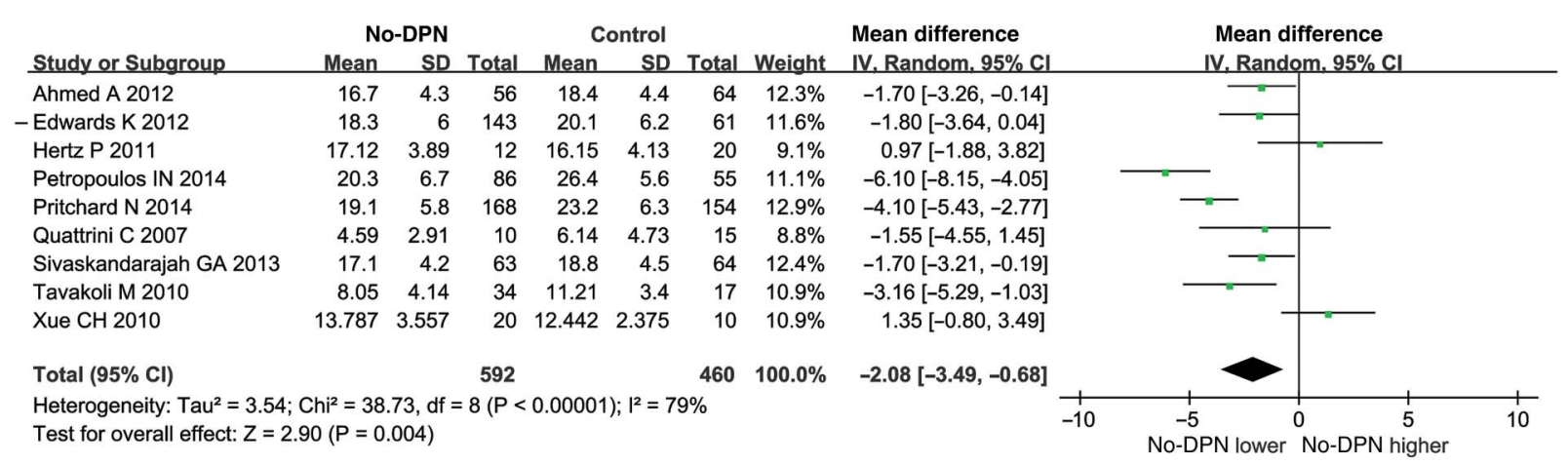

Figure 4 Forest plots of nerve fibre length (NFL) test results in diabetic peripheral neuropathy (DPN), healthy control and no-DPN groups.

There was substantial between-study heterogeneity $(\mathrm{p}<0.05$ and $\left.\mathrm{I}^{2}>50 \%\right)$. To assess possible explanations for the heterogeneity, we first applied single-factor meta-regression analysis by adding the number of patients, publication year, mean age, male-female distribution and duration time of diabetes separately as variates. A statistical difference was only found when adding the number of patients as a variate $(p=0.04)$. Subgroup analysis was then executed based on the type of CCM (laser-scanning confocal microscope, LSCM vs slit-scanning confocal microscope, SSCM), on the age balance setting (matched age vs no matched age) and on the papers where the person interpreting the outcome was masked to the DPN status (masked vs no masked). No significant difference was found $(p>0.05)$. The detailed results of subgroup analysis are depicted in online supplementary table S1 and figures S1-S9. To further assess the reliability of our results, we performed a sensitivity analysis by sequentially excluding individual studies. Statistically similar results were obtained after sequentially excluding each study, suggesting stability of the meta-analysis. Funnel plots of these CCM parameters showed mild asymmetry visually, suggesting a publication bias.

\section{DISCUSSION}

DPN, characterised by chronic paresthaesia and electrophysiological abnormalities, is the most common chronic complication of diabetes mellitus. ${ }^{22}$ The cornea, owing to its transparent property, allows direct, non-invasive and in vivo imaging of the small unmyelinated nerve fibre bundle with CCM. The application of CCM in imaging the cornea provides a new approach to the study of corneal nerve morphology. Studies in diabetic patients have shown that corneal nerve damage assessed with CCM relates to the severity of intraepidermal nerve fibre loss in foot skin biopsies. ${ }^{13} \mathrm{~A}$ further significant potential of CCM has been demonstrated with CCM detecting nerve fibre regeneration after simultaneous pancreas and kidney transplantation ${ }^{10}$ before symptoms, clinical neurologic deficits, nerve conduction attributes, corneal sensations and even skin biopsy. Our meta-analysis confirmed that corneal nerve changes could be detected using CCM in patients with DPN by detecting that NFD, NBD and NFL were significantly reduced in patients with DPN compared with healthy controls and diabetic patients without DPN. However, the current study found no significant differences in TCs between the DPN and control group or DPN 
and no-DPN group. In fact, controversy has existed about TC changes in DPN patients, with TC having been reported as increased, ${ }^{8} 17$ reduced $^{10} 16$ or unchanged. ${ }^{5-7} \quad 14$ In our meta-analysis, although not statistically significant, the pooled mean TC value in the DPN group was higher than the healthy controls (WMD $=0.22)$ and no-DPN patients (WMD $=0.15$ ). Experiments have demonstrated increased tortuosity of regenerating nerves, particularly in older animals. ${ }^{23}$ Thus, increased tortuosity could be a morphologic marker of regeneration. Patient characteristics, such as age, duration of diabetes or glycaemic control in diabetic patients, and study design, such as techniques or equipment used in the studies, may also influence TC changes. Furthermore, impairment in corneal nerve parameters also correlated with the severity of neuropathy. In the current study, we discussed the variety of severity gradings reported in the included studies.

Meta-regression analyses, subgroup analysis, sensitivity analysis and creation of funnel plots were performed to assess between-study heterogeneity $\left(\mathrm{p}<0.05\right.$ and $\left.\mathrm{I}^{2}>50 \%\right)$. Meta-regression analyses showed that publication year, mean age, male-female distribution and duration of diabetes had no significant influence on heterogeneity except for the number of patients $(p=0.04)$. Subgroup analysis confirmed that the type of CCM (LSCM vs SSCM) and age balance setting showed no statistical differences. Funnel plots with mild asymmetry suggested a publication bias. Except for those discussed above, there may still be variables that might differ among studies, such as the severity of neuropathy of the cohorts examined, the acquisition mode with CCM, the number of images analysed per participant, the field of view of the acquisition lens, operator technique and software applied to analyse images. Because of variation in data presentation or incomplete reporting of data, the effect of these variables on between-study heterogeneity could not be further examined. Another limitation was that there are multiple ways of defining morphological parameters and no consensus has been reached regarding 'gold standard' definitions of parameters. Finally, for practical reasons, the eligible studies only covered those that were written in English and Chinese, which might bring bias. The funnel plot for publication bias was indeed significant, although this result may also indicate small-study effects; that is, effects in small studies may be larger than effects in larger studies. The meta-regression test also proved that the sample size had an impact on heterogeneity. Therefore, the results of the funnel plot could be attributed to small-study effects. Further research is required to analyse this.

In conclusion, our meta-analysis was based on a larger sample size compared with the individual studies. The results demonstrated that NFD, NBD and NFL were significantly reduced in patients with DPN compared with controls and diabetic patients without DPN, while the changes in TCs were not significant. Assessment of corneal nerve morphology with CCM could be a promising method for diagnosing and evaluating diabetic patients with DPN.

Contributors All authors declare that they have participated sufficiently in the conception and design of this work or the analysis and interpretation of the data, and the writing of the manuscript to take public responsibility for it. Neither this manuscript nor one with substantially similar content under our authorship has been published or is being considered for publication elsewhere.

Funding This work was supported by grants from the National Key Foundation for Exploring Scientific Instrument of China (2013YQ03065104), the National Science and Technology Major Project of the Ministry of Science and Technology of China (2014ZX07104), the National Science and Technology support program of China (2012BAI23B00) and the National Natural Science Foundation of China (81402461).

Competing interests None.

Provenance and peer review Not commissioned; externally peer reviewed.

\section{REFERENCES}

1 Tesfaye S, Boulton AJ, Dyck PJ, et al. Diabetic neuropathies: update on definitions, diagnostic criteria, estimation of severity, and treatments. Diabetes Care 2010;33:2285-93.

2 Papanas N, Ziegler D. Corneal confocal microscopy: a new technique for early detection of diabetic. Curr Diab Rep 2013;13:488-99.

3 Tavakoli M, Petropoulos IN, Malik RA. Assessing corneal nerve structure and function in diabetic neuropathy. Clin Exp Optom 2012;95:338-47.

4 Tavakoli M, Petropoulos IN, Malik RA. Corneal confocal microscopy to assess diabetic neuropathy: an eye on the foot. J Diabetes Sci Technol 2013;7:1179-89.

5 Ahmed A, Bril V, Orszag A, et al. Detection of diabetic sensorimotor polyneuropathy by corneal confocal microscopy in type 1 diabetes: a concurrent validity study. Diabetes Care 2012;35:821-8.

6 Edwards K, Pritchard N, Vagenas D, et al. Utility of corneal confocal microscopy for assessing mild diabetic neuropathy: baseline findings of the LANDMark study. Clin Exp Optom 2012:95:348-54.

7 Hertz P, Bril V, Orszag A, et al. Reproducibility of in vivo corneal confocal microscopy as a novel screening test for early diabetic sensorimotor polyneuropathy. Diabet Med 2011;28:1253-60.

8 Kallinikos P, Berhanu M, O'Donnell C, et al. Corneal nerve tortuosity in diabetic patients with neuropathy. Investig Ophthalmol Vis Sci. 2004;45:418-22.

9 Malik RA, Kallinikos P, Abbott CA, et al. Corneal confocal microscopy: a non-invasive surrogate of nerve fibre damage and repair in diabetic patients. Diabetologia 2003;46:683-8.

10 Mehra S, Tavakoli M, Kallinikos PA, et al. Corneal confocal microscopy detects early nerve regeneration after pancreas transplantation in patients with type 1 diabetes. Diabetes Care 2007;30:2608-12.

11 Petropoulos IN, Alam U, Fadavi H, et al. Rapid automated diagnosis of diabetic peripheral neuropathy with in vivo corneal confocal microscopy. Invest Ophthalmol Vis Sci 2014;55:2071-8.

12 Pritchard N, Edwards K, Dehghani C, et al. Longitudinal assessment of neuropathy in type 1 diabetes using novel ophthalmic markers (LANDMark): study design and baseline characteristics. Diabetes Res Clin Pract 2014;104:248-56.

13 Quattrini C, Tavakoli M, Jeziorska M, et al. Surrogate markers of small fiber damage in human diabetic neuropathy. Diabetes 2007;56:2148-54.

14 Sivaskandarajah GA, Halpern EM, Lovblom LE, et al. Structure-function relationship between corneal nerves and conventional small-fiber tests in type 1 diabetes. Diabetes Care 2013;36:2748-55.

15 Tavakoli M, Quattrini C, Abbott C, et al. Corneal confocal microscopy: a novel noninvasive test to diagnose and stratify the severity of human diabetic neuropathy. Diabetes Care 2010;33:1792-7.

16 Tavakoli M, Kallinikos P, Iqbal A, et al. Corneal confocal microscopy detects improvement in corneal nerve morphology with an improvement in risk factors for diabetic neuropathy. Diabet Med 2011;28:1261-7.

17 Xue CH, Shao JQ, Lu CW, et al. Early diagnosis of peripheral neuropathy by corneal confocal microscopy. Zhongguo Tang Niao Bing Za Zhi 2010;18:679-81.

18 Tavakoli M, Boulton AJ, Efron N, et al. Increased Langerhans cell density and corneal nerve damage in diabetic patients: role of immune mechanisms in human diabetic neuropathy. Cont Lens Anterior Eye 2011;34:7-11.

19 Petropoulos IN, Alam U, Fadavi $\mathrm{H}$, et al. Corneal nerve loss detected with corneal confocal microscopy is symmetrical and related to the severity of diabetic polyneuropathy. Diabetes Care 2013;36:3646-51.

20 Higgins JPT, Green S. eds. Cochrane Handbook for Systematic Reviews of Interventions 2011. The Cochrane Collaboration; Version 5.1.0 [updated March 2011], 2011. http://www.cochrane-handbook.org

21 Moher D, Liberati A, Tetzlaff J, et al. Preferred reporting items for systematic reviews and meta-analyses: the PRISMA statement. Ann Intern Med 2009;151:264-9, W64.

22 Solomon T, Dinesh S. Advances in the epidemiology, pathogenesis and management of diabetic peripheral neuropathy. Diabetes Metab Res Rev 2012;28 (Suppl 1):8-14.

23 Kawabuchi M, Chongjian Z, Islam AT, et al. The effect of aging on the morphological nerve changes during muscle reinnervation after nerve crush. Restor Neurol Neurosci 1998;13:117-27. 